\title{
Genome sequence of the ocean sediment bacterium Saccharomonospora marina type strain $\left(\mathrm{XMU15}{ }^{\mathrm{T}}\right)$
}

\author{
Hans-Peter Klenk ${ }^{1^{*}}$, Megan Lu' ${ }^{2}$, Susan Lucas ${ }^{3}$, Alla Lapidus ${ }^{3}$, Alex Copeland ${ }^{3}$, Sam Pitluck ${ }^{3}$, \\ Lynne A. Goodwin ${ }^{2,3}$, Cliff Han ${ }^{2,3}$, Roxanne Tapia ${ }^{2,3}$, Evelyne-Marie Brambilla ${ }^{1}$, Gabriele \\ Pötter $^{1}$, Miriam Land ${ }^{3,4}$, Natalia Ivanova ${ }^{3}$, Manfred Rohde ${ }^{5}$, Markus Göker ${ }^{1}$, John C. \\ Detter $^{2,3}$, Wen-Jun Li ${ }^{6}$, Nikos C. Kyrpides ${ }^{3}$, and Tanja Woyke ${ }^{3}$ \\ ${ }^{1}$ Leibniz Institute DSMZ - German Collection of Microorganisms and Cell Cultures, \\ Braunschweig, Germany \\ ${ }^{2}$ Los Alamos National Laboratory, Bioscience Division, Los Alamos, New Mexico, USA \\ ${ }^{3}$ DOE Joint Genome Institute, Walnut Creek, California, USA \\ ${ }^{4}$ Oak Ridge National Laboratory, Oak Ridge, Tennessee, USA \\ ${ }^{5}$ HZI - Helmholtz Centre for Infection Research, Braunschweig, Germany \\ ${ }^{6}$ Yunnan Institute of Microbiology, Yunnan University, Kunming, China \\ *Corresponding author: Hans-Peter Klenk
}

Keywords: aerobic, chemoheterotrophic, Gram-positive, vegetative and aerial mycelia, spore-forming, non-motile, marine bacterium, Pseudonocardiaceae, CSP 2010

Saccharomonospora marina Liu et al. 2010 is a member of the genus Saccharomonospora, in the family Pseudonocardiaceae that is poorly characterized at the genome level thus far. Members of the genus Saccharomonospora are of interest because they originate from diverse habitats, such as leaf litter, manure, compost, surface of peat, moist, over-heated grain, and ocean sediment, where they might play a role in the primary degradation of plant material by attacking hemicellulose. Organisms belonging to the genus are usually Gram-positive staining, non-acid fast, and classify among the actinomycetes. Here we describe the features of this organism, together with the complete genome sequence (permanent draft status), and annotation. The 5,965,593 bp long chromosome with its 5,727 protein-coding and 57 RNA genes was sequenced as part of the DOE funded Community Sequencing Program (CSP) 2010 at the Joint Genome Institute (JGI).

\section{Introduction}

Strain $\mathrm{XMU15}^{\mathrm{T}}$ (= DSM $45390=$ KCTC $19701=$ CCTCC AA 209048) is the type strain of the species Saccharomonospora marina [1], one of nine species currently in the genus Saccharomonospora [2]. The strain was originally isolated from an ocean sediment sample collected from Zhaoan Bay, East China Sea, in 2005 [1]. The genus name Saccharomonospora was derived from the Greek words for sakchâr, sugar, monos, single or solitary, and spore, a seed or spore, meaning the sugar (containing) single-spored (organism) [3]; the species epithet was derived from the Latin adjective marina, of the sea, referring to the origin of the strain [1]. S. marina and the other type strains of the genus Saccharomonospora were selected for genome sequencing in one of the DOE Community Sequencing Projects (CSP 312) at Joint Genome Institute (JGI), because members of the genus (which originate from diverse habitats, such as leaf litter, manure, compost, surface of peat, moist, over-heated grain and ocean sediment) might play a role in the primary degradation of plant material by attacking hemicellulose. This expectation was underpinned by the results of the analysis of the genome of $S$. viridis [4], one of the recently sequenced GEBA genomes [5]. The $S$. viridis genome, the first sequenced genome from the genus Saccharomonospora, contained an unusually large 
number (24 in total) genes for glycosyl hydrolases (GH) belonging to $14 \mathrm{GH}$ families, which were identified in the Carbon Active Enzyme Database [6]. Hydrolysis of cellulose and starch were also reported for other members of the genus (that are included in CSP 312), including S. marina [1], S. halophila [7], S. saliphila [8], S. paurometabolica [9], and S. xinjiangensis [10]. Here we present a summary classification and a set of features for $S$. marina XMU15 ${ }^{\mathrm{T}}$, together with the description of the genomic sequencing and annotation.

\section{Classification and features}

A representative genomic $16 \mathrm{~S}$ rRNA sequence of $S$. marina XMU15 ${ }^{\mathrm{T}}$ was compared using NCBI BLAST $[11,12]$ under default settings (e.g., considering only the high-scoring segment pairs (HSPs) from the best 250 hits) with the most recent release of the Greengenes database [13] and the relative frequencies of taxa and keywords (reduced to their stem [14]) were determined, weighted by BLAST scores. The most frequently occurring genera were Gordonia (63.5\%), Saccharomonospora (24.1\%), Actinomycetospora (4.5\%), Actinopolyspora (1.8\%) and Pseudonocardia (1.4\%) (195 hits in total). Regarding the single hit to sequences from members of the species, the average identity within HSPs was 99.7\%, whereas the average coverage by HSPs was $100.1 \%$. Regarding the 23 hits to sequences from other members of the genus, the average identity within HSPs was 96.1\%, whereas the average coverage by HSPs was $98.3 \%$. Among all other species, the one yielding the highest score was Saccharomonospora saliphila (HM368568), which corresponded to an identity of $99.9 \%$ and an HSP coverage of $92.1 \%$. (Note that the Greengenes database uses the INSDC (= EMBL/NCBI/DDBJ) annotation, which is not an authoritative source for nomenclature or classification. For instance, the Gordonia hits are likely to be caused by misannotations in INSDC). The highest-scoring environmental sequence was FN667533 ('stages composting process pilot scale municipal drum compost clone PS3734'), which showed an identity of $96.0 \%$ and a HSP coverage of $97.9 \%$. The most frequently occurring keywords within the labels of all environmental samples which yielded hits were 'skin' (6.3\%), 'forearm' (2.8\%), 'soil' (2.6\%), 'fossa' $(2.5 \%)$ and 'volar' (2.3\%) (55 hits in total). These keywords do not fit to the known habitat of strain $\mathrm{XMU}^{\mathrm{T}}$, because Saccharomonospora rarely occurs in environmental samples so that more distant relatives (here from human skin) distort the automatically generated list of keywords. Environmental samples which yielded hits of a higher score than the highest scoring species were not found.

Figure 1 shows the phylogenetic neighborhood of $S$. marina in a $16 \mathrm{~S}$ rRNA based tree. The sequences of the three 16S rRNA gene copies in the genome differ from each other by up to 13 nucleotides, and differ by up to 15 nucleotides from the previously published 16S rRNA sequence (FJ812357).

Cells of $S$. marina XMU15 ${ }^{\mathrm{T}}$ are non-acid fast, stain Gram-positive and form an irregularly branched vegetative mycelium of 0.3 to $0.4 \mu \mathrm{m}$ diameter (Figure 2) [1]. Non-motile, smooth or wrinkled spores were observed on the aerial mycelium, occasionally in short spore chains [1]. The growth range of strain $\mathrm{XMU} 15^{\mathrm{T}}$ spans from $28-37^{\circ} \mathrm{C}$, with an optimum at $28^{\circ} \mathrm{C}$, and pH 7.0 on ISP 2 medium [1]. Strain $\mathrm{XMU}^{\mathrm{T}} 5^{\mathrm{T}}$ grows well in up to $5 \% \mathrm{NaCl}$, with an optimum at $0-3 \% \mathrm{NaCl}$ [1]. Substrates used by the strain are summarized in the strain description [1].

\section{Chemotaxonomy}

The cell wall of strain XMU15 ${ }^{\mathrm{T}}$ contains mesodiaminopimelic acid [1]; arabinose, galactose and ribose are present [1]. The fatty acids spectrum is dominated by penta- to heptadecanoic acids: iso- $\mathrm{C}_{16: 0}$ (26.4\%), $\mathrm{C}_{17: 1 \text { шбс }}(16.8 \%), \mathrm{C}_{16: 0}$ (palmitic acid, 8.9\%), $\mathrm{C}_{15: 0}(16.2 \%), \mathrm{C}_{17: 1 \text { w8c }}(7.7 \%)$, iso- $\mathrm{C}_{16: 1 \text { н }}(6.0 \%)$ [1]. Main menaquinone is MK-9 $\mathrm{H}_{4}(90 \%)$ complemented by MK-8 $\mathrm{H}_{4}$ (10\%) [1]; phospholipids comprised phosphatidylglycerol, diphosphatidylglycerol, and phosphatidylinisitol with a minor fraction of phosphatidylethanolamine [1].

\section{Genome sequencing and annotation Genome project history}

This organism was selected for sequencing as part of the DOE Joint Genome Institute Community Sequencing Program (CSP) 2010, CSP 312, "Whole genome type strain sequences of the genus Saccharomonospora - a taxonomically troubled genus with bioenergetic potential". The genome project is deposited in the Genomes On Line Database [21] and the complete genome sequence is deposited in GenBank. Sequencing, finishing and annotation were performed by the DOE Joint Genome Institute (JGI). A summary of the project information is shown in Table 2. 


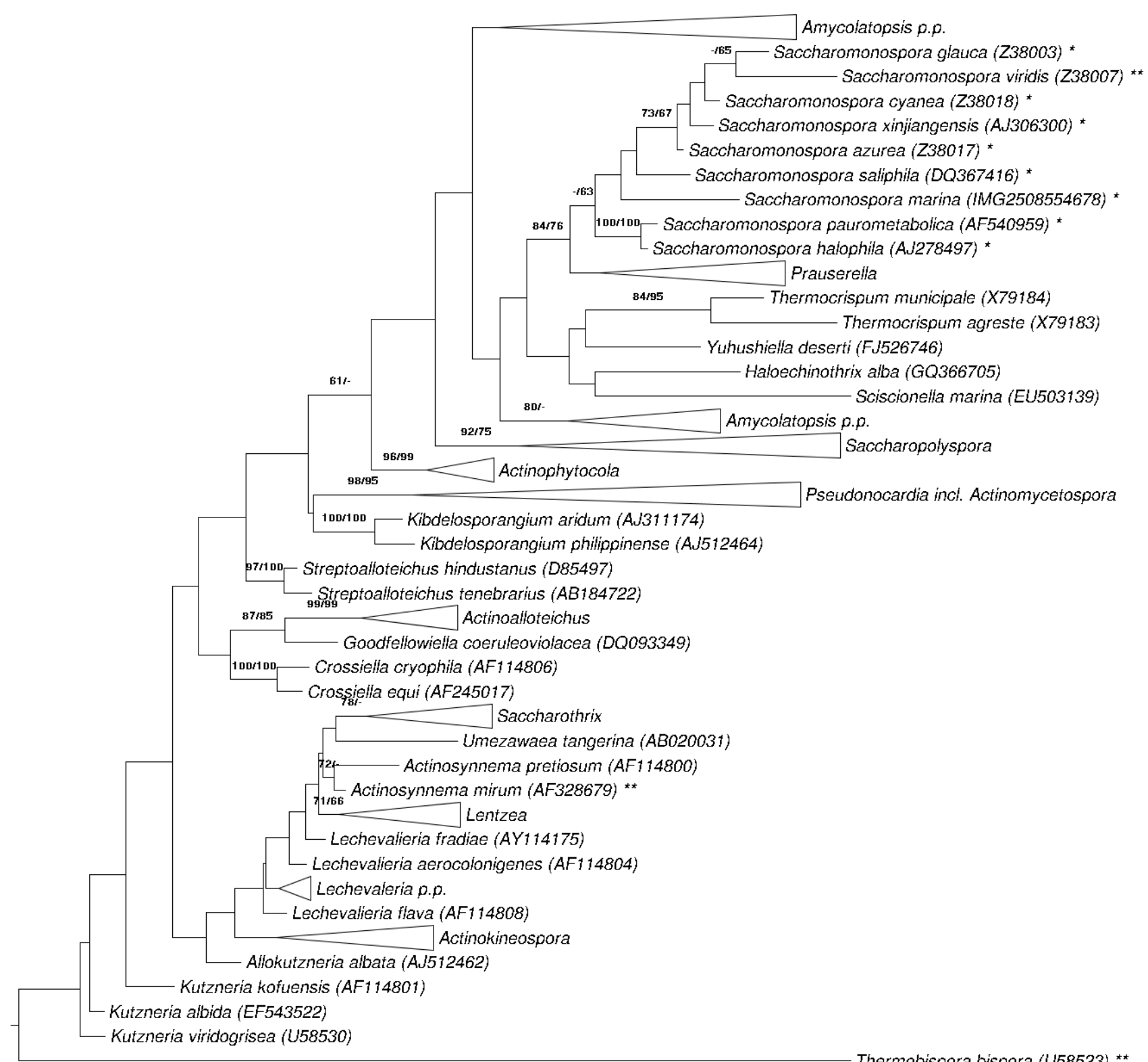

0.05

Figure 1. Phylogenetic tree highlighting the position of $S$. marina relative to the type strains of the other species within the family Pseudonocardiaceae. The tree was inferred from 1,391 aligned characters $[15,16]$ of the $16 \mathrm{~S}$ rRNA gene sequence under the maximum likelihood (ML) criterion [17]. Rooting was done initially using the midpoint method [18] and then checked for its agreement with the current classification (Table 1). The branches are scaled in terms of the expected number of substitutions per site. Numbers adjacent to the branches are support values from $600 \mathrm{ML}$ bootstrap replicates [19] (left) and from 1,000 maximum-parsimony bootstrap replicates [20] (right) if larger than 60\%. Lineages with type strain genome sequencing projects registered in GOLD [21] are labeled with one asterisk, those also listed as 'Complete and Published' with two asterisks $[4,22,23]$, with $S$. azurea missing second asterisk but published in this issue [24]. Actinopolyspora iraqiensis Ruan et al. 1994 [25] was ignored in the tree. The species was proposed to be a later heterotypic synonym of $S$. halophila [26], although the name $A$. iraqiensis would have had priority over $S$. halophila. This taxonomic problem will soon be resolved with regard to the genomes of $A$. iraqiensis and S. halophila, which were both part of CSP 312 . 


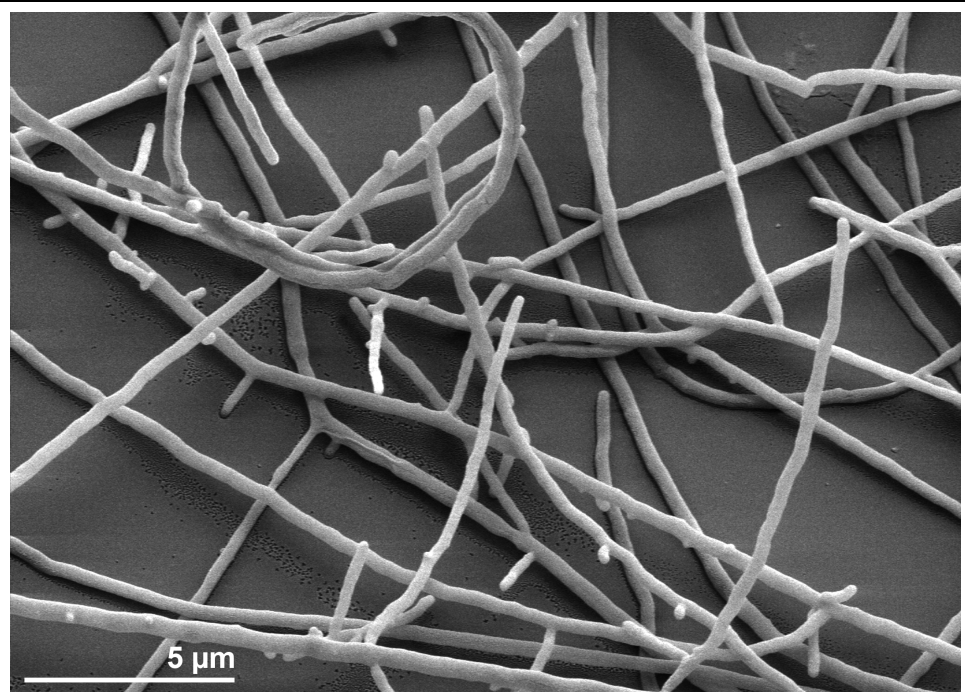

Figure 2. Scanning electron micrograph of S. marina XMU15 ${ }^{\top}$

Table 1. Classification and general features of S. marina XMU15 ${ }^{\top}$ according to the MIGS recommendations [27].

\begin{tabular}{|c|c|c|c|}
\hline MIGS ID & Property & Term & Evidence code \\
\hline & \multirow{10}{*}{ Current classification } & Domain Bacteria & TAS [28] \\
\hline & & Phylum Actinobacteria & TAS [29] \\
\hline & & Class Actinobacteria & TAS [30] \\
\hline & & Subclass Actinobacteridae & TAS $[30,31]$ \\
\hline & & Order Actinomycetales & TAS [30-33] \\
\hline & & Suborder Pseudonocardineae & TAS $[30,31,34]$ \\
\hline & & Family Pseudonocardiaceae & TAS $[30,31,34-36]$ \\
\hline & & Genus Saccharomonospora & TAS $[32,37]$ \\
\hline & & Species Saccharomonospora marina & TAS [1] \\
\hline & & Type-strain XMU15 & TAS [1] \\
\hline & Gram stain & positive & TAS [1] \\
\hline & Cell shape & variable, substrate and aerial mycelia & TAS [1] \\
\hline & Motility & non-motile & TAS [1] \\
\hline & Sporulation & $\begin{array}{l}\text { smooth or wrinkled spores, singly, in pairs or in short } \\
\text { chains from aerial mycelium }\end{array}$ & TAS [1] \\
\hline & Temperature range & mesophile & TAS [1] \\
\hline & Optimum temperature & $28-37^{\circ} \mathrm{C}$ & TAS [1] \\
\hline & Salinity & optimum $0-3 \%(\mathrm{w} / \mathrm{v}) \mathrm{NaCl}$, tolerated up to $5 \%$ & TAS [1] \\
\hline \multirow[t]{3}{*}{ MIGS-22 } & Oxygen requirement & aerobic & TAS [1] \\
\hline & Carbon source & D-glucose, manose, melibiose, L-rhamnose, myo-inositol & TAS [1] \\
\hline & Energy metabolism & chemoheterotrophic & NAS \\
\hline MIGS-6 & Habitat & marine, ocean sediment & TAS [1] \\
\hline MIGS-15 & Biotic relationship & free living & TAS [1] \\
\hline \multirow{2}{*}{ MIGS-14 } & Pathogenicity & none & NAS \\
\hline & Biosafety level & 1 & NAS \\
\hline MIGS-23.1 & Isolation & ocean sediment & TAS [1] \\
\hline MIGS-4 & Geographic location & Zhaoan Bay, East China Sea & TAS [1] \\
\hline MIGS-5 & Sample collection time & December 2005 & NAS \\
\hline MIGS-4.1 & Latitude & 24.108 & TAS [1] \\
\hline MIGS-4.2 & Longitude & 117.294 & TAS [1] \\
\hline MIGS-4.3 & Depth & $4 \mathrm{~m}$ & TAS [1] \\
\hline MIGS-4.4 & Altitude & $-4 \mathrm{~m}$ & TAS [1] \\
\hline
\end{tabular}

Evidence codes - IDA: Inferred from Direct Assay (first time in publication); TAS: Traceable Author Statement (i.e., a direct report exists in the literature); NAS: Non-traceable Author Statement (i.e., not directly observed for the living, isolated sample, but based on a generally accepted property for the species, or anecdotal evidence). These evidence codes are from the Gene Ontology project. If the evidence code is IDA, then the property was directly observed for a living isolate by one of the authors or an expert mentioned in the acknowledgements [38]. 
Klenk et al.

\begin{tabular}{lll}
\hline \multicolumn{2}{l}{ Table 2. Genome sequencing project information } \\
\hline MIGS ID & Property & Term \\
\hline MIGS-31 & Finishing quality & Permanent draft \\
MIGS-28 & Libraries used & Three genomic libraries: one 454 pyrosequence standard library, one \\
& & 454 PE library (10 kb insert size), one Illumina library \\
MIGS-29 & Sequencing platforms & Illumina GAii, 454 GS FLX Titanium \\
MIGS-31.2 & Sequencing coverage & 780.0 $~$ Illumina; 8.6 × pyrosequence \\
MIGS-30 & Assemblers & Newbler version 2.3, Velvet version 1.0.13, phrap version SPS - 4.24 \\
MIGS-32 & Gene calling method & Prodigal \\
& INSDC ID & CM001439 \\
& GenBank Date of Release & February 3, 2012 \\
& GOLD ID & Gi07581 \\
& NCBI project ID & 61991 \\
& Database: IMG & 2508501012 \\
MIGS-13 & Source material identifier & DSM 45390 \\
& Project relevance & Bioenergy and phylogenetic diversity \\
\hline
\end{tabular}

\section{Growth conditions and DNA isolation}

The history of strain XMU15 ${ }^{\mathrm{T}}$ starts in 2005 with an isolate from ocean sediment collected from Zhaoan Bay in the East China Sea, followed by a detailed chemotaxonomic description by Liu et al. [1], and deposit of the strain in three collections in 2009: Korean Collection for Type Cultures (accession 19701), Chinese Centre for Type Cultures Collections (accession 209048) and German Collection of Microorganisms and Cell cultures, DSMZ (accession 45390). Strain XMU15T, DSM 45390, was grown in DSMZ medium 83 (Czapek Peptone Medium) [39] at $28^{\circ} \mathrm{C}$. DNA was isolated from 0.5$1 \mathrm{~g}$ of cell paste using Jetflex Genomic DNA Purification Kit (GENOMED 600100) following the standard protocol as recommended by the manufacturer with the following modifications: extended cell lysis time (60 min.) with additional $30 \mu \mathrm{l}$ achromopeptidase, lysostaphin, mutanolysin; proteinase $\mathrm{K}$ was added at 6 -fold the supplier recommended amount for $60 \mathrm{~min}$. at $58^{\circ} \mathrm{C}$. The purity, quality and size of the bulk gDNA preparation were assessed by JGI according to DOE-JGI guidelines. DNA is available through the DNA Bank Network [40].

\section{Genome sequencing and assembly}

The genome was sequenced using a combination of Illumina and 454 sequencing platforms. All general aspects of library construction and sequencing can be found at the JGI website [41]. Pyrosequencing reads were assembled using the Newbler assembler (Roche). The initial Newbler assembly consisting of 185 contigs in one scaffold was converted into a phrap [42] assembly by making fake reads from the consensus, to collect the read pairs in the 454 paired end library. Illumina GAii sequencing data $(5,096.2 \mathrm{Mb})$ was assembled with Velvet [43] and the consensus sequences were shredded into $1.5 \mathrm{~kb}$ overlapped fake reads and assembled together with the 454 data. The 454 draft assembly was based on $95.6 \mathrm{Mb} 454$ draft data and all of the 454 paired end data. Newbler parameters are -consed -a 50 -1 350 -g -m -ml 20. The Phred/Phrap/Consed software package [42] was used for sequence assembly and quality assessment in the subsequent finishing process. After the shotgun stage, reads were assembled with parallel phrap (High Performance Software, LLC). Possible mis-assemblies were corrected with gapResolution [41], Dupfinisher [44], or sequencing cloned bridging PCR fragments with subcloning. Gaps between contigs were closed by editing in Consed, by PCR and by Bubble PCR primer walks (J.-F. Chang, unpublished). A total of 233 additional reactions were necessary to close gaps and to raise the quality of the finished sequence. Illumina reads were also used to correct potential base errors and increase consensus quality using a software Polisher developed at JGI [45]. The error rate of the completed genome sequence is less than 1 in 100,000. Together, the combination of the Illumina and 454 sequencing platforms provided $788.6 \times$ coverage of the genome. The final assembly contained 397,729 pyrosequence and 61,582,867 Illumina reads. 


\section{Genome annotation}

Genes were identified using Prodigal [46] as part of the Oak Ridge National Laboratory genome annotation pipeline, followed by a round of manual curation using the JGI GenePRIMP pipeline [47]. The predicted CDSs were translated and used to search the National Center for Biotechnology Information (NCBI) non-redundant database, UniProt, TIGRFam, Pfam, PRIAM, KEGG, COG, and InterPro databases. These data sources were combined to assert a product description for each predicted protein. Non-coding genes and miscellaneous features were predicted using tRNAscanSE [48], RNAMMer [49], Rfam [50], TMHMM [51], and signalP [52].

\section{Genome properties}

The genome consists of a 5,965,593 bp long circular chromosome with a $68.9 \% \mathrm{G}+\mathrm{C}$ content (Table 3 and Figure 3). Of the 5,784 genes predicted, 5,727 were protein-coding genes, and 57 RNAs; 149 pseudogenes were also identified. The majority of the protein-coding genes $(75.0 \%)$ were assigned a putative function while the remaining ones were annotated as hypothetical proteins. The distribution of genes into COGs functional categories is presented in Table 4.

Table 3. Genome Statistics

\begin{tabular}{lrr}
\hline Attribute & Value & \% of Total \\
\hline Genome size (bp) & $5,965,593$ & $100.00 \%$ \\
DNA coding region (bp) & $5,364,872$ & $89.93 \%$ \\
DNA G+C content (bp) & $4,112,466$ & $68.94 \%$ \\
Number of replicons & 1 & \\
Extrachromosomal elements & 0 & \\
Total genes & 5,784 & $100.00 \%$ \\
RNA genes & 57 & $0.99 \%$ \\
rRNA operons & 3 & \\
tRNA genes & 47 & $0.81 \%$ \\
Protein-coding genes & 5,727 & $99.01 \%$ \\
Pseudo genes & 149 & $2.58 \%$ \\
Genes with function prediction (proteins) & 4,341 & $75.05 \%$ \\
Genes in paralog clusters & 3,491 & $60.36 \%$ \\
Genes assigned to COGs & 4,261 & $73.67 \%$ \\
Genes assigned Pfam domains & 4,426 & $76.52 \%$ \\
Genes with signal peptides & 1,159 & $20.04 \%$ \\
Genes with transmembrane helices & 1,256 & $21.72 \%$ \\
CRISPR repeats & 1 & \\
\hline
\end{tabular}




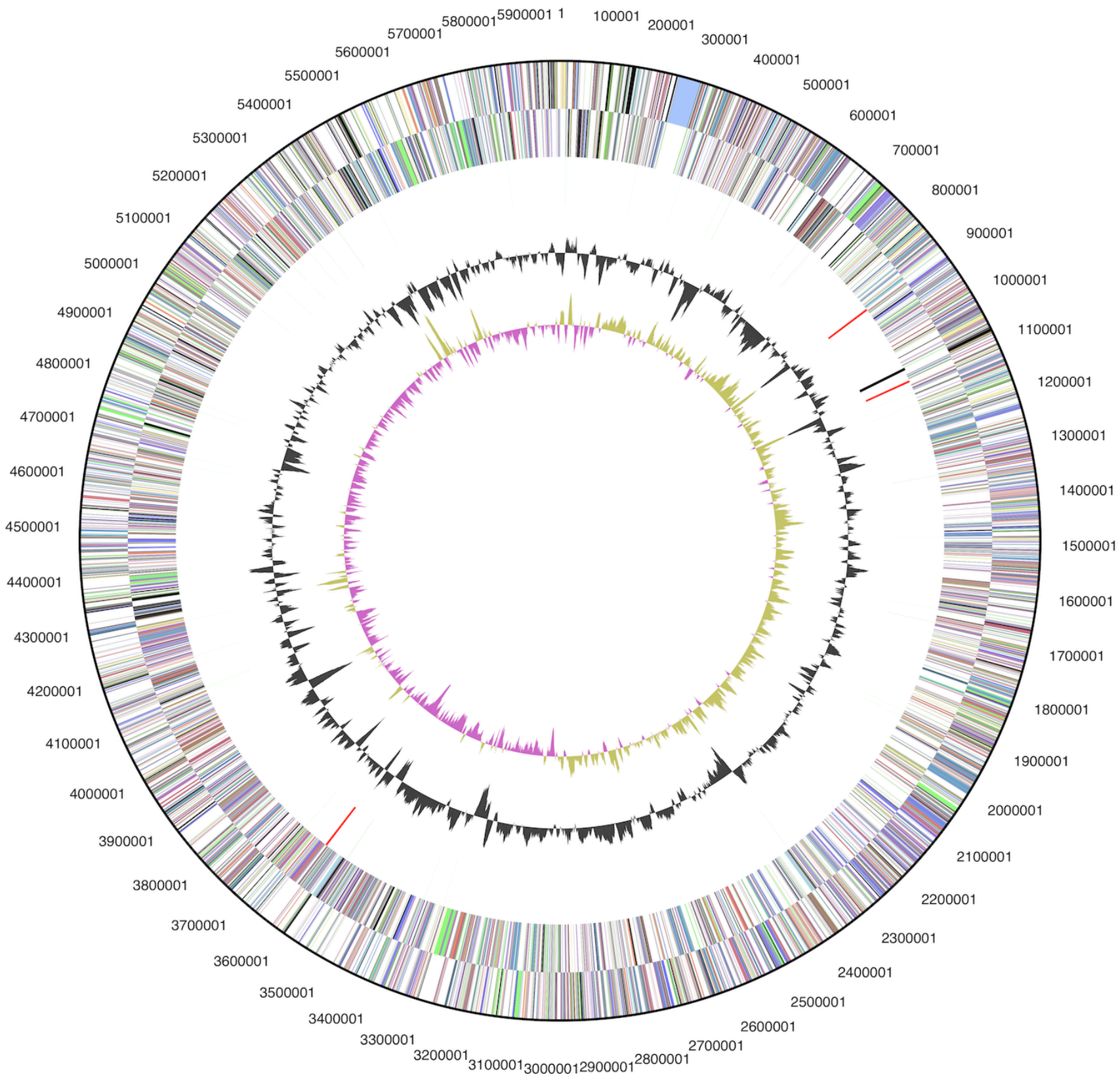

Figure 3. Graphical map of the chromosome. From outside to the center: Genes on forward strand (color by COG categories), Genes on reverse strand (color by COG categories), RNA genes (tRNAs green, rRNAs red, other RNAs black), GC content, GC skew. 
Table 4. Number of genes associated with the general COG functional categories

\begin{tabular}{crrl}
\hline Code & value & \%age & Description \\
\hline J & 173 & 3.6 & Translation, ribosomal structure and biogenesis \\
A & 3 & 0.1 & RNA processing and modification \\
K & 509 & 10.7 & Transcription \\
L & 226 & 4.7 & Replication, recombination and repair \\
B & 3 & 0.1 & Chromatin structure and dynamics \\
D & 40 & 0.8 & Cell cycle control, cell division, chromosome partitioning \\
Y & 0 & 0.0 & Nuclear structure \\
V & 68 & 1.4 & Defense mechanisms \\
T & 220 & 4.6 & Signal transduction mechanisms \\
M & 191 & 4.0 & Cell wall/membrane biogenesis \\
N & 6 & 0.1 & Cell motility \\
Z & 0 & 0.0 & Cytoskeleton \\
W & 0 & 0.0 & Extracellular structures \\
U & 52 & 1.1 & Intracellular trafficking and secretion, and vesicular transport \\
O & 152 & 3.2 & Posttranslational modification, protein turnover, chaperones \\
C & 369 & 7.7 & Energy production and conversion \\
G & 294 & 6.2 & Carbohydrate transport and metabolism \\
E & 381 & 8.0 & Amino acid transport and metabolism \\
F & 93 & 2.0 & Nucleotide transport and metabolism \\
H & 223 & 4.7 & Coenzyme transport and metabolism \\
I & 291 & 6.1 & Lipid transport and metabolism \\
P & 210 & 4.4 & Inorganic ion transport and metabolism \\
Q & 264 & 5.5 & Secondary metabolites biosynthesis, transport and catabolism \\
R & 649 & 13.6 & General function prediction only \\
S & 364 & 7.6 & Function unknown \\
- & 1,523 & 26.4 & Not in COGs \\
\hline & & &
\end{tabular}

\section{Acknowledgements}

The work conducted by the U.S. Department of Energy Joint Genome Institute was supported by the Office of
Science of the U.S. Department of Energy under Contract No. DE-AC02-05CH11231.

\section{References}

1. Liu Z, Li Y, Zheng LQ, Huang YJ, Li WJ.

Saccharomonospora marina sp. nov., isolated from ocean sediment of the East China Sea. Int J Syst Evol Microbiol 2010; 60:1854-1857. PubMedhttp://dx.doi.org/10.1099/ijs.0.017038-0

2. Garrity G. NamesforLife. BrowserTool takes expertise out of the database and puts it right in the browser. Microbiol Today 2010; 37:9.

3. Euzéby JP. List of Bacterial Names with Standing in Nomenclature: a folder available on the internet. Int J Syst Bacteriol 1997; 47:590. Pub-

Medhttp://dx.doi.org/10.1099/00207713-47-2$\underline{590}$

4. Pati A, Sikorski J, Nolan M, Lapidus A, Copeland A, Glavina Del Rio T, Lucas S, Chen F, Tice H, Pitluck S, et al. Complete genome sequence of Saccharomonospora viridis type strain $\left(\mathrm{P} 101^{\top}\right)$. Stand Genomic Sci 2009; 1:141-149. PubMedhttp://dx.doi.org/10.4056/sigs.20263

5. Wu D, Hugenholtz P, Mavromatis K, Pukall R, Dalin E, Ivanova NN, Kunin V, Goodwin L, Wu 
Klenk et al.

M, Tindall BJ, et al. A phylogeny-driven Genomic Encyclopaedia of Bacteria and Archaea. Nature 2009; 462:1056-1060. PubMedhttp://dx.doi.org/10.1038/nature08656

6. Carbon Active Enzyme Database. www.cazy.org.

7. Al-Zarban SS, Al-Musallam AA, Abbas I, Stackebrandt E, Kroppenstedt RM.

Saccharomonospora halophila sp. nov., a novel halophilic actinomycete isolated from marsh soil in Kuwait. Int / Syst Evol Microbiol 2002; 52:555558. PubMed

8. Syed DG, Tang SK, Cai M, Zhi XY, Agasar D, Lee JC, Kim CJ, Jiang CL, Xu LH, Li WJ.

Saccharomonospora saliphila sp. nov., a halophilic actinomycete from an Indian soil. Int J Syst Evol Microbiol 2008; 58:570-573. PubMedhttp://dx.doi.org/10.1099/ijs.0.65449-0

9. Li WJ, Tang SK, Stackebrandt E, Kroppenstedt RM, Schumann P, Xu LH, Jiang CL.

Saccharomonospora paurometabolica sp. nov., a moderately halophilic actinomycete isolated from soil in China. Int I Syst Evol Microbiol 2003;

53:1591-1594. PubMedhttp://dx.doi.org/10.1099/ijs.0.02633-0

10. Jin X, Xu LH, Mao PH, Hseu TH, Jiang CL. Description of Saccharomonospora xinjiangensis sp. nov. based on chemical and molecular classification. Int J Syst Bacteriol 1998; 48:1095-1099. PubMedhttp://dx.doi.org/10.1099/00207713-484-1095

11. Altschul SF, Gish W, Miller W, Myers EW, Lipman DJ. Bascic local alignment search tool. J Mol Biol 1990; 215:403-410. PubMed

12. Korf I, Yandell M, Bedell J. BLAST, O'Reilly, Sebastopol, 2003.

13. DeSantis TZ, Hugenholtz $P$, Larsen $N$, Rojas $M$, Brodie EL, Keller K, Huber T, Dalevi D, Hu P, Andersen GL. Greengenes, a chimera-checked $16 \mathrm{~S}$ rRNA gene database and workbench compatible with ARB. Appl Environ Microbiol 2006; 72:5069-5072. PubMedhttp://dx.doi.org/10.1128/AEM.03006-05

14. Porter MF. An algorithm for suffix stripping. Program: electronic library and information systems 1980; 14:130-137.

15. Lee C, Grasso C, Sharlow MF. Multiple sequence alignment using partial order graphs. BioinformatiCS 2002; 18:452-464. PubMedhttp://dx.doi.org/10.1093/bioinformatics/18.3 $\underline{.452}$
16. Castresana J. Selection of conserved blocks from multiple alignments for their use in phylogenetic analysis. Mol Biol Evol 2000; 17:540-552. PubMedhttp://dx.doi.org/10.1093/oxfordjournals.mol bev.a026334

17. Stamatakis A, Hoover P, Rougemont J. A rapid bootstrap algorithm for the RAxML web servers. Syst Biol 2008; 57:758-771. PubMedhttp://dx.doi.org/10.1080/106351508024296 $\underline{42}$

18. Hess PN, De Moraes Russo CA. An empirical test of the midpoint rooting method. Biol / Linn SoC Lond 2007; 92:669674.http://dx.doi.org/10.1111/j.10958312.2007.00864.x

19. Pattengale ND, Alipour M, Bininda-Emonds ORP, Moret BME, Stamatakis A. How many bootstrap replicates are necessary? Lect Notes Comput Sci 2009; 5541:184-

200.http://dx.doi.org/10.1007/978-3-642-02008$\underline{7 \_13}$

20. Swofford DL. PAUP*: Phylogenetic Analysis Using Parsimony (*and Other Methods), Version 4.0 b10. Sinauer Associates, Sunderland, 2002.

21. Liolios K, Chen IM, Mavromatis K, Tavernarakis $\mathrm{N}$, Kyrpides NC. The genomes on line database (GOLD) in 2009: Status of genomic and metagenomic projects and their associated metadata. Nucleic Acids Res 2010; 38:D346D354. PubMedhttp://dx.doi.org/10.1093/nar/gkp848

22. Land M, Lapidus A, Mayilraj S, Chen R, Copeland A, Glavina Del Rio T, Nolan M, Lucas S, Tice H, Cheng JF, et al. Complete genome sequence of Actinosynnema mirum type strain $\left(101^{\mathrm{T}}\right)$. Stand Genomic Sci 2009; 1:46-53. PubMedhttp://dx.doi.org/10.4056/sigs.21137

23. Liolios K, Sikorski J, Jando M, Lapidus A, Copeland A, Glavina Del Rio T, Nolan M, Lucas $\mathrm{S}$, Tice $\mathrm{H}$, Cheng JF, et al. Complete genome sequence of Thermobispora bispora type strain $\left(\mathrm{R} 51^{\mathrm{T}}\right)$. Stand Genomic Sci 2010; 2:318-326. PubMedhttp://dx.doi.org/10.4056/sigs.962171

24. Klenk HP, Held B, Lucas S, Lapidus A, Copeland A, Hammon N, Pitluck S, Goodwin LA, Han C, Tapia R, et al. Genome sequence of the soil bacterium Saccharomonospora azurea type strain (NA-128 $8^{\top}$ ). Stand Genomic Sci 2012; (this issue).

25. Ruan SJ, Al-Tai AM, Zhou ZH, Qu LH. Actinopolyspora iraqiensis sp. nov., a new halophilic actinomycete isolated from soil. Int J Syst Bacteriol 1994; 44:759- 
763.http://dx.doi.org/10.1099/00207713-44-4$\underline{759}$

26. Tang SK, Wang Y, Klenk HP, Shi R, Lou K, Zhang YJ, Chen C, Ruan JS, Li WJ. Actinopolyspora alba sp. nov. and Actinopolyspora erythraea sp. nov., isolated from a salt field, and reclassification of Actinopolyspora iraqiensis Ruan et al. 1994 as a heterotypic synonym of Saccharomonospora halophila. Int J Syst Evol Microbiol 2011;

61:1693-1698. PubMedhttp://dx.doi.org/10.1099/ijs.0.022319-0

27. Field D, Garrity G, Gray T, Morrison N, Selengut J, Sterk P, Tatusova T, Thomson N, Allen MJ, Angiuoli SV, et al. The minimum information about a genome sequence (MIGS) specification. Nat Biotechnol 2008; 26:541-547. PubMedhttp://dx.doi.org/10.1038/nbt1360

28. Woese CR, Kandler O, Wheelis ML. Towards a natural system of organisms. Proposal for the domains Archaea and Bacteria. Proc Natl Acad Sci USA 1990; 87:4576-4579. PubMedhttp://dx.doi.org/10.1073/pnas.87.12.4576

29. Garrity GM, Holt JG. The Road Map to the Manual. In: Garrity GM, Boone DR, Castenholz RW (eds), Bergey's Manual of Systematic Bacteriology, Second Edition, Volume 1, Springer, New York, 2001, p. 119-169.

30. Stackebrandt E, Rainey FA, Ward-Rainey NL. Proposal for a new hierarchic classification system, Actinobacteria classis nov. Int I Syst Bacteriol 1997; 47:479-

491.http://dx.doi.org/10.1099/00207713-47-2$\underline{479}$

31. Zhi XY, Li WJ, Stackebrandt E. An update of the structure and 16S rRNA gene sequence-based definition of higher ranks of the class Actinobacteria, with the proposal of two new suborders and four new families and emended descriptions of the existing higher taxa. Int I Syst Evol Microbiol 2009; 59:589-608. PubMedhttp://dx.doi.org/10.1099/ijs.0.65780-0

32. Skerman VBD, McGowan V, Sneath PHA. Approved Lists of Bacterial Names. Int J Syst Bacteriol 1980; 30:225-

420.http://dx.doi.org/10.1099/00207713-30-1$\underline{225}$

33. Buchanan RE. Studies in the nomenclature and classification of bacteria. II. The primary subdivisions of the Schizomycetes. J Bacteriol 1917; 2:155-164. PubMed

34. Labeda DP, Goodfellow M, Chun J, Zhi X-Y, Li $W J$. Reassessment of the systematics of the subor- der Pseudonocardineae: transfer of the genera within the family Actinosynnemataceae Labeda and Kroppenstedt 2000 emend. Zhi et al. 2009 into an emended family Pseudonocardiaceae Embley et al. 1989 emend. Zhi et al. 2009. Int J Syst Evol Microbiol 2011; 61:1259-1264. PubMedhttp://dx.doi.org/10.1099/ijs.0.024984-0

35. List Editor. Validation List no. 29. Validation of the publication of new names and new combinations previously effectively published outside the IJSB. Int J Syst Bacteriol 1989; 39:205206.http://dx.doi.org/10.1099/00207713-39-2$\underline{205}$

36. Embley MT, Smida J, Stackebrandt E. The phylogeny of mycolate-less wall chemotype IV Actinomycetes and description of Pseudonocardiaceae fam. nov. Syst Appl Microbiol 1988; 11:4452.http://dx.doi.org/10.1016/S07232020(88)80047-X

37. Nonomura $\mathrm{H}$, Ohara Y. Distribution of actinomycetes in soil. X. New genus and species of monosporic actinomycetes in soil. J Ferment Technol 1971; 49:895-903.

38. Ashburner M, Ball CA, Blake JA, Botstein D, Butler H, Cherry JM, Davis AP, Dolinski K, Dwight SS, Eppig JT, et al. Gene ontology: tool for the unification of biology. The Gene Ontology Consortium. Nat Genet 2000; 25:25-29. PubMedhttp://dx.doi.org/10.1038/75556

39. List of growth media used at DSMZ. http://www.dsmz.de/catalogues/cataloguemicroorganisms/culture-technology/list-of-mediafor-microorganisms.html.

40. Gemeinholzer B, Dröge $G$, Zetzsche $H$, Haszprunar G, Klenk HP, Güntsch A, Berendsohn WG, Wägele JW. The DNA Bank Network: the start from a German initiative. Biopreserv Biobank $2011 ; 9: 51$ -

55.http://dx.doi.org/10.1089/bio.2010.0029

41. The DOE Joint Genome Institute. www.jgi.doe.gov

42. Phrap and Phred for Windows. MacOS, Linux, and Unix. www.phrap.com

43. Zerbino DR, Birney E. Velvet: algorithms for de novo short read assembly using de Bruijn graphs. Genome Res 2008; 18:821-829. PubMedhttp://dx.doi.org/10.1101/gr.074492.107

44. Han C, Chain P. Finishing repeat regions automatically with Dupfinisher. In: Proceeding of the 2006 international conference on bioinformatics 
Klenk et al.

\& computational biology. Arabnia HR, Valafar $\mathrm{H}$ (eds), CSREA Press. June 26-29, 2006: 141-146.

45. Lapidus A, LaButti K, Foster B, Lowry S, Trong S, Goltsman E. POLISHER: An effective tool for using ultra short reads in microbial genome assembly and finishing. AGBT, Marco Island, FL, 2008.

46. Hyatt $\mathrm{D}$, Chen $\mathrm{GL}$, Locascio PF, Land ML, Larimer FW, Hauser LJ. Prodigal Prokaryotic Dynamic Programming Genefinding Algorithm. BMC Bioinformatics 2010; 11:119. PubMedhttp://dx.doi.org/10.1186/1471-2105-11-119

47. Pati A, Ivanova N, Mikhailova N, Ovchinikova G, Hooper SD, Lykidis A, Kyrpides NC. GenePRIMP: A Gene Prediction Improvement Pipeline for microbial genomes. Nat Methods 2010; 7:455-457. PubMedhttp://dx.doi.org/10.1038/nmeth.1457

48. Lowe TM, Eddy SR. tRNAscan-SE: a program for improved detection of transfer RNA genes in genomic sequence. Nucleic Acids Res 1997;

25:955-964. PubMed
49. Lagesen K, Hallin PF, Rødland E, Stærfeldt HH, Rognes T, Ussery DW. RNammer: consistent annotation of rRNA genes in genomic sequences. Nucleic Acids Res 2007; 35:3100-3108. PubMedhttp://dx.doi.org/10.1093/nar/gkm160

50. Griffiths-Jones S, Bateman A, Marshall M, Khanna A, Eddy SR. Rfam: an RNA family database. Nucleic Acids Res 2003; 31:439-441. PubMedhttp://dx.doi.org/10.1093/nar/gkg006

51. Krogh A, Larsson B, von Heijne G, Sonnhammer ELL. Predicting transmembrane protein topology with a hidden Markov model: Application to complete genomes. J Mol Biol 2001; 305:567580. PubMedhttp://dx.doi.org/10.1006/jmbi.2000.4315

52. Bendtsen JD, Nielsen H, von Heijne G, Brunak S. Improved prediction of signal peptides: SignalP 3.0. J Mol Biol 2004; 340:783-795. PubMedhttp://dx.doi.org/10.1016/j.jmb.2004.05.028 